Original Research Paper

\title{
Surface Level Estimator-An Automated Contouring Instrument
}

\author{
Varun Sai Chinthala and Gajendra Dixit \\ Department of Mechanical Engineering, Maulana Azad National Institute of Technology, Bhopal, India
}

\section{Article history}

Received: 21-04-2017

Revised: 22-07-2017

Accepted: 29-08-2017

Corresponding Author: Gajendra Dixit

Department of Mechanical Engineering, Maulana Azad

National Institute of Technology,

Bhopal, India

Email: dixitgajendra1@gmail.com

\begin{abstract}
This paper introduces a dynamic electronic instrument to measure the inclination of a surface, named as Surface Level Estimator (SLE). This device is designed to reduce the human effort and time consumed for civil surveying. This paper describes the construction, working principle, programming and experimentation results of the instrument. Finally, the conclusion and scope of future is discussed at the end of the paper.
\end{abstract}

Keywords: Surveying, Surface Level Estimator, Arduino Uno, Levelling

\section{Introduction}

The object of surveying is to prepare a plan or map so that it represents the area on a horizontal plane, vertical distances between the points are shown by contour lines or some other methods, vertical sections are usually represented by means of vertical sections drawn separately (Chrzanowski, 2014; Jetko, 2011).

Levelling is a branch of surveying, the object of which is (a) To find the elevations of points with respect to a given or assumed datum, which is required to enable the works to be designed and (b) to establish points at a given elevation or at different elevations with respect to a given or assumed datum, which is required in the setting out of all kind of engineering works. levelling deals with measurements in the vertical plane (Xeidakis, 1994).

A contour is defined as an imaginary line of constant elevation on the ground surface. It is the science of representing the vertical dimension of the terrain on a two-dimensional map. The now existing instruments regarding this point of surface levelling are Hand level, Abney clinometer, Indian pattern clinometer, burnel hand level, De Lisle's clinometer, theodolite etc (Kyle, 1983; Ustinova et al., 2012).

The foresaid methods require large human efforts, time and good working condition of instrument for an optimised result of work, in order to overcome all these barriers, an automated electronic device is designed to reduce human power and time, simultaneously increasing the efficiency of the work.

Surface Level Estimator (SLE) is an automated and dynamic electronic device, which traverse the surface to be surveyed and records inclinations of the section of a surface at various equal intervals with respect to a fore considered datum. Through which the contour lines of the area could be easily plotted. It provides work comfort by decreasing a lot of human effort and can give precise results about the surface to be surveyed.

\section{Principle}

The axis of gravity will always act towards the centre of the earth, the shift in the axis of gravity with respect to considered datum can always be expressed in terms of inclinations.

\section{Construction and Working}

The Surface level estimator is a dynamic instrument which traces over the land to be surveyed, the movement of the instrument is obtained from a simple motor drive.

Potentiometer, Rotary encoder and Arduino are connected as shown in the Fig. 1. Various parts of the instrument are (a) Rotary encoder (b) Potentiometer (c) Arduino Uno (d) Weights (e) Connecting Wires (f) Switch (g) Resistor.

The device travels on the path to be surveyed. The motion of the device is provided by a simple motor drive. A weight is hanged to a rod (yellow colored rod shown in Fig. 2). Axis1 shown in Fig. 2 is a fixed axis. Axis2 shown in Fig. 2 carries a weight and is freely allowed to oscillate and is always directed to the centre of the earth. The angle between the Axis1 and Axis2 $\left(\theta_{1}\right)$ varies according to the inclination of the surface over which the SLE travels. As the device moves on the surface, according to the inclination of the surface, the 
angle $\theta_{1}$ changes. The axis 2 is connected to the nib of the potentiometer such that the movement of axis2 creates the rotary motion of the nib of the potentiometer. The potentiometer is connected to a voltage source and a setup to record the output of potentiometer. Thereby the output voltage of the potentiometer changes according to the rotary motion of nib of the potentiometer and the voltage readings are recorded. The readings are recorded using a microcontroller based on ATmega328P, Arduino Uno.

Axis 2 is always perpendicular to the horizontal plane ( $\theta 2$ equals to 90 degrees as shown in Fig. 2). As the inclination of the surface changes, the angle $\theta 1$ changes accordingly, the potentiometer is attached to the axis 2 in such a way that, if there is a $\theta$ angle change between axis 1 and axis2, then the potentiometer also rotates by same angle $\theta$. Hence the output voltage from the potentiometer changes according to the angle $\theta$, which implies that the output voltage of a potentiometer depends on the inclination of the surface. Hence this implies that for every angle of inclination we have a corresponding value of voltage output.

The device is programmed in such a way that for every unit distance the device travels (distance is calculated from the rotary encoder attached to one of the wheel) the output voltage of the potentiometer is recorded, which corresponds to the inclination of the surface at that point (Harris et al., 1997).

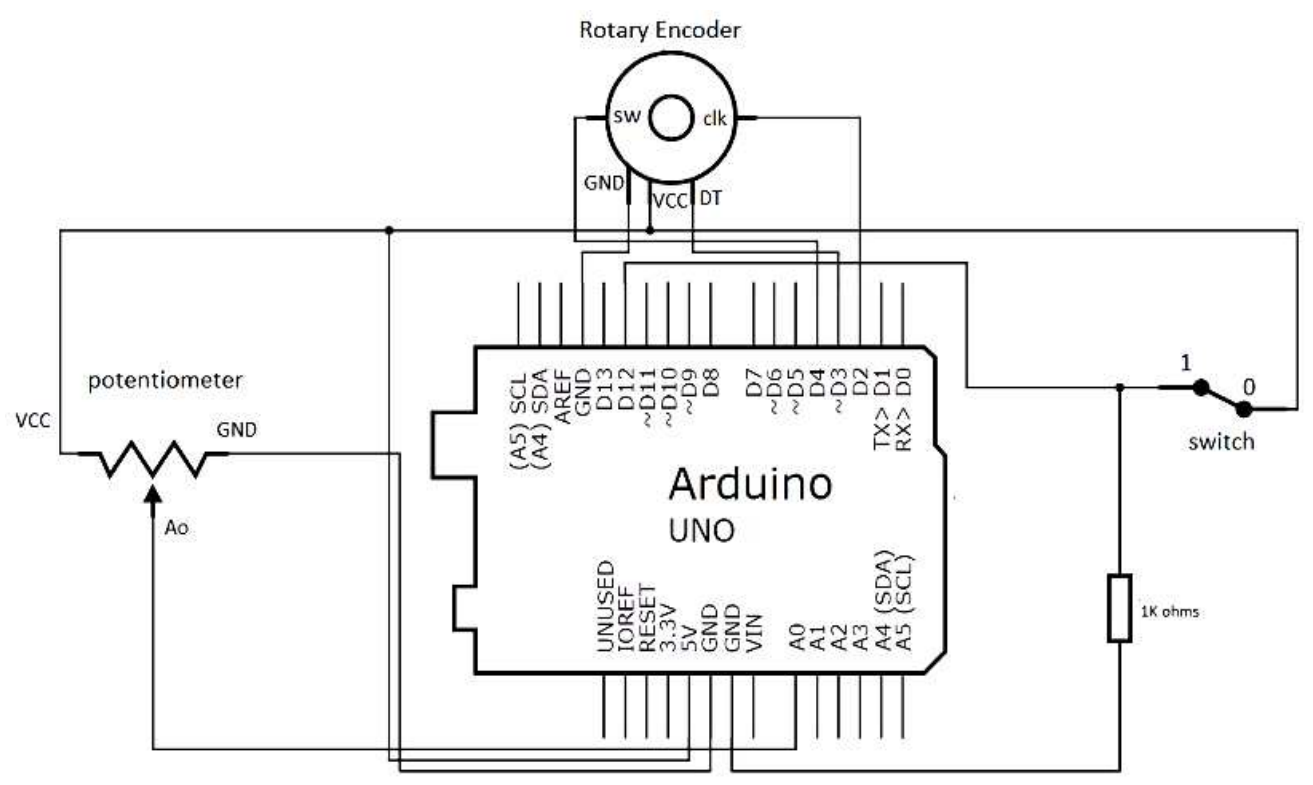

Fig. 1. The electronic circuit of surface level estimator

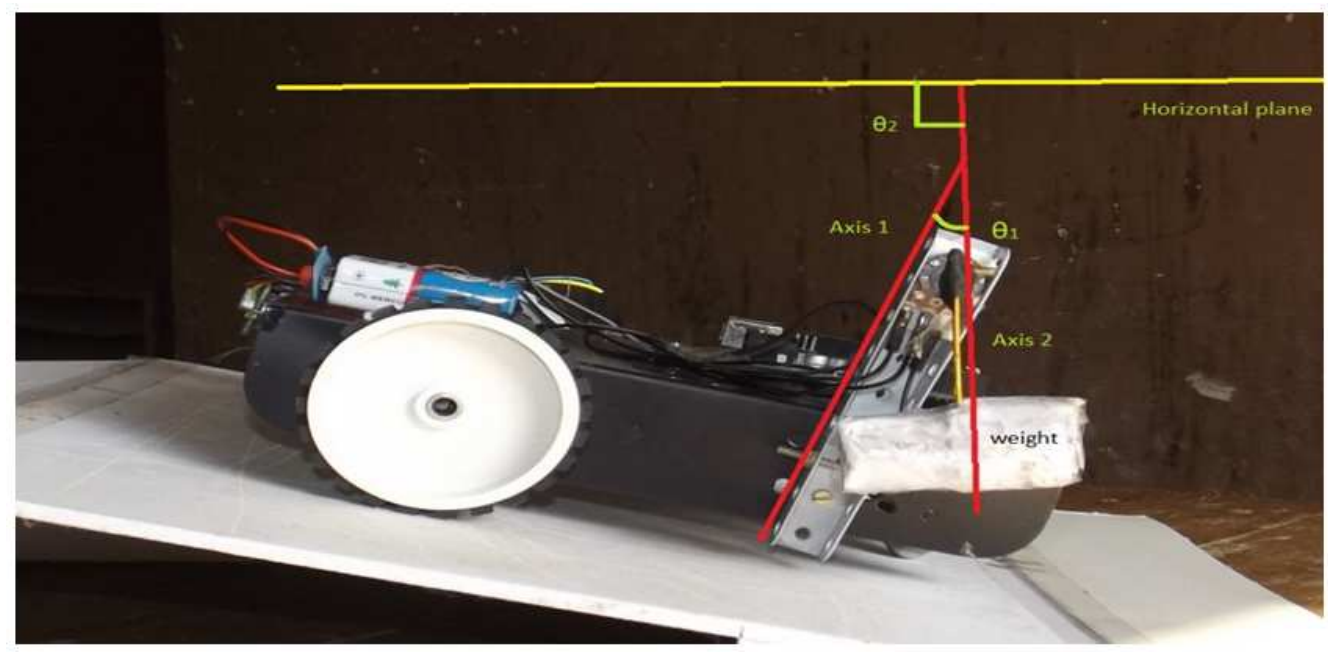

Fig. 2. Axis1, Axis2, $\theta_{1}$ and $\theta_{2}$, are shown on SLE 


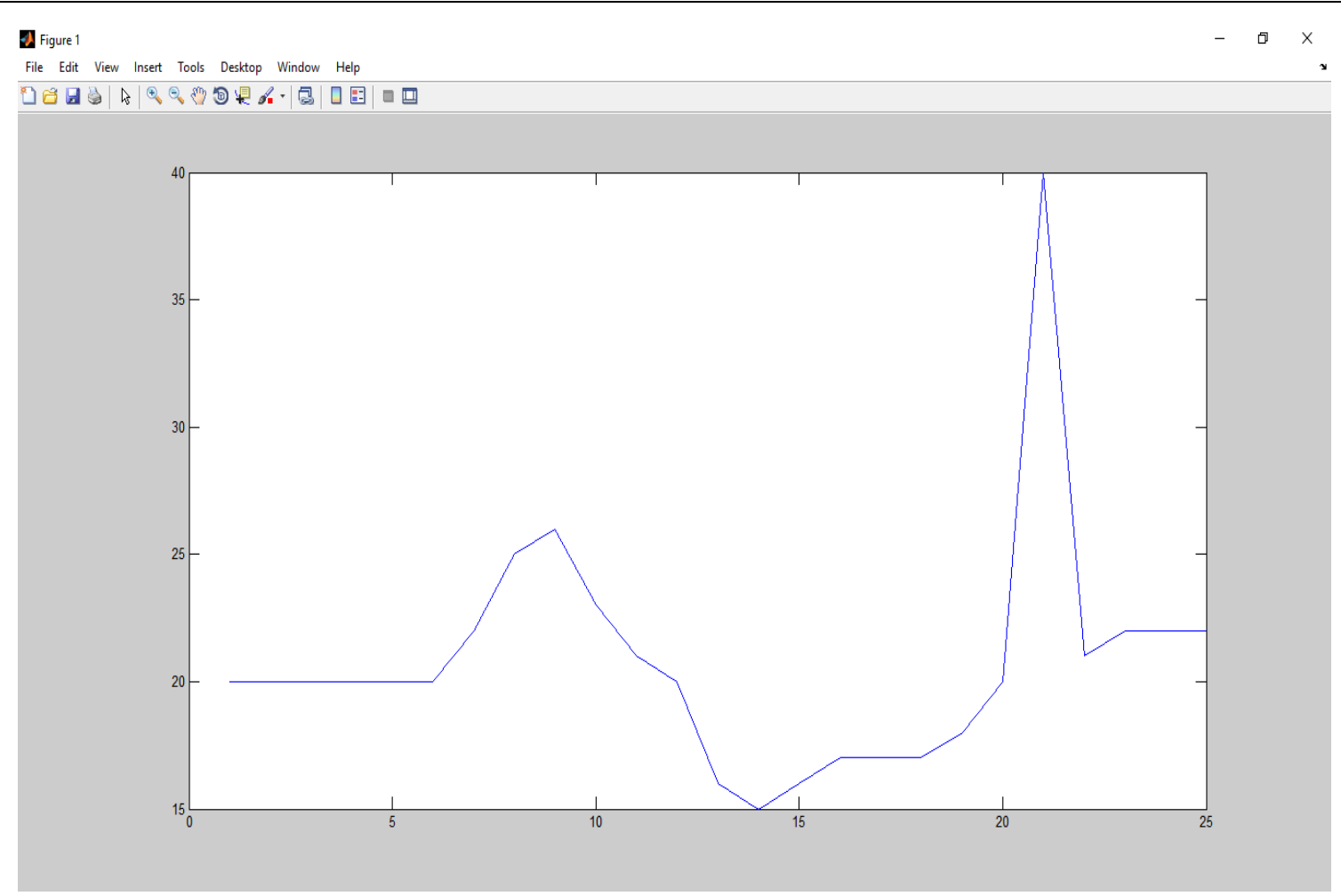

Fig. 3. The output values plotted in a graph using MATLAB

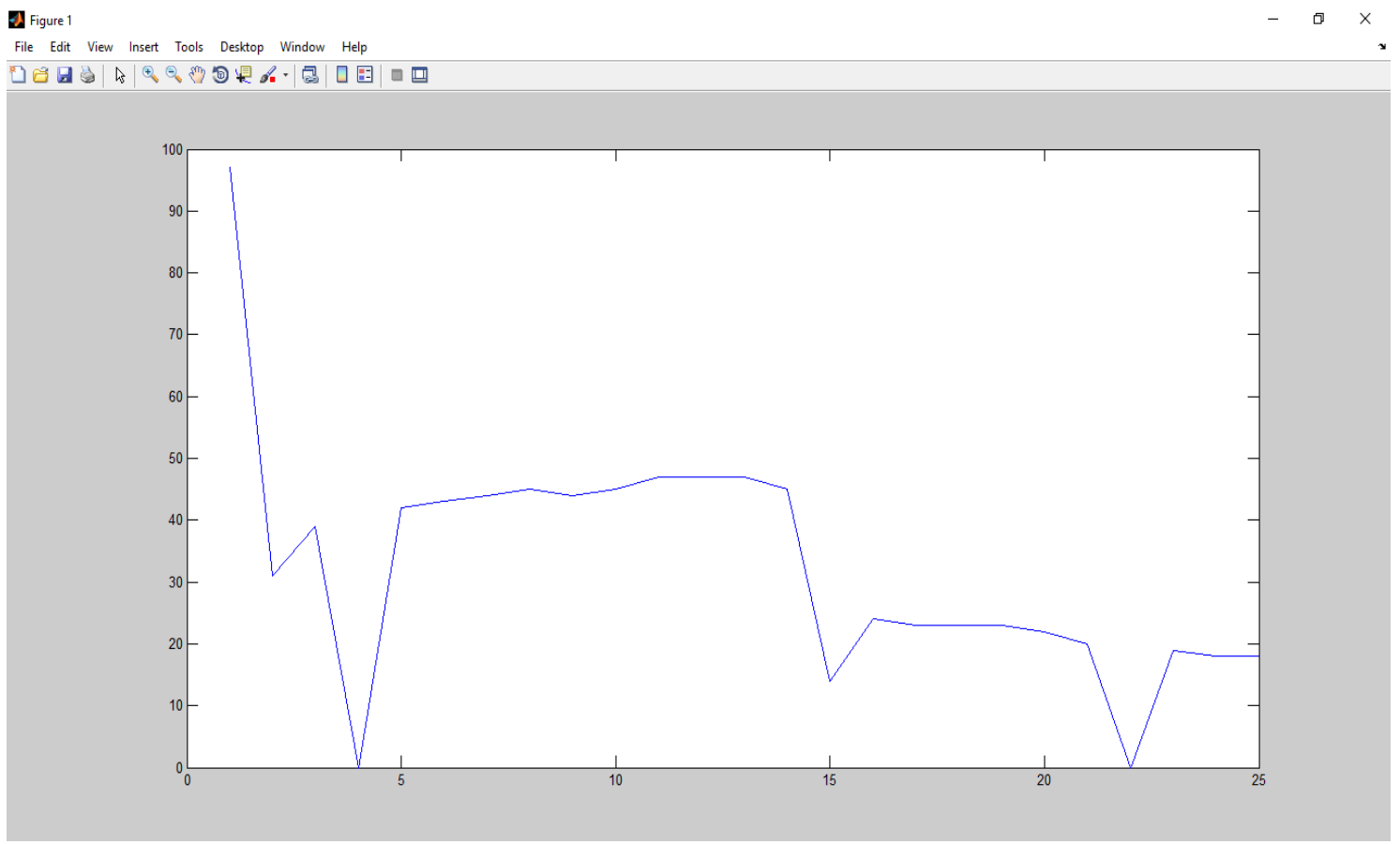

Fig. 4. The output values plotted in a graph using MATLAB

The device is programmed in such a way that when the device moves on the surface, the readings are recorded and are used to plot graphs (shown in Fig. 3 and 4. in MATLAB. The output of the device is plotted in form of graphs to analyse the inclinations of the surface at different points. 


\section{Program used in Arduino}

The device is programmed in such a way that switch shown in Fig. 1 should be open when the device moves on the surface and during which the readings are recorded and the switch should be closed when the readings stored are used to plot graphs in MATLAB.

\section{$/ *$ surface level estimator program*/}

\#include $<$ EEPROM.h $>$

const int $\mathrm{clkPin}=2 ; / /$ the clk pin attach to pin 2

const int $\mathrm{dtPin}=3 ; / /$ the dt pin attach to pin 3

const int $\operatorname{swPin}=4 ; / /$ the sw pin attach to pin 4

int addr $=1$;

int complete $=0$;

int encoderVal $=0$;

int sensorPin $=\mathrm{A} 0 ; / /$ select the input pin for the potentiometer

void $\operatorname{setup}()$

\{

//set clkPin,dePin,swPin as INPUT

pinMode(clkPin, INPUT);

pinMode(12,INPUT) ;

pinMode(13,OUTPUT) ;

pinMode(dtPin, INPUT);

pinMode(swPin, INPUT);

digitalWrite(swPin, HIGH);

Serial.begin(9600); // initialize serial communications at 9600 bps

\}

void $\operatorname{loop}()$

\{

int rtpin = digitalRead(12);

if $($ complete $!=1 \& \&$ rtpin $==$ LOW $)$

\{

int change $=$ getEncoderTurn( $) ; / /$

encoderVal $=$ encoderVal + change;

Serial.println(map(analogRead(sensorPin),0,1023,0,40));

if $($ encoderVal\%10 $==0 \& \&$ encoderVal $<141)\{$

EEPROM.write(encoderVal/10,map(analogRead(sensor Pin), 0, 1023,0,40)) ;

\}

if (encoderVal $>141)\{$

complete $=1$;

digitalWrite(13, HIGH); // turn the LED on (HIGH is the voltage level)

\}

\}

if $($ rtpin $==$ HIGH) \{

Serial.println(EEPROM.read(addr));

addr++;

\}

int getEncoderTurn(void)

\{

static int oldA = HIGH; //set the oldA as HIGH static int oldB $=\mathrm{HIGH}$;/set the oldB as $\mathrm{HIGH}$

int result $=0$;

int newA = digitalRead(clkPin);//read the value of clkPin to newA

int newB $=$ digitalRead(dtPin);//read the value of $\mathrm{dtPin}$ to newB

if (newA != oldA $\mid$ newB != oldB)//if the value of clkPin or the dtPin has changed

\{

// something has changed

if $($ oldA $==\mathrm{HIGH} \& \&$ newA $==\mathrm{LOW})$

\{

result $=($ oldB $* 2-1)$;

\}

\}

old $\mathrm{A}=$ newA;

oldB $=$ newB;

return result;

\}

Program for MATLAB

clear all

clc

arduino = serial('COM3','BaudRate',9600);

fopen(arduino);

$\mathrm{x}=1: 10$

$\mathrm{y}=\mathrm{x}$

for $\mathrm{i}=1$ :length $(\mathrm{x})$

$y(i)=$ fscanf(arduino, $\left.{ }^{\prime} \% d^{\prime}\right)$;

pause(1);

end

fclose(arduino);

disp('making plot..')

plot(x,y);

clear arduino;

\section{Calibration of the Instrument}

Firstly, the device is tested on the surface of known inclinations and the voltage outputs of potentiometer are recorded for the various angles of inclinations. Every angle of inclinations corresponds to a particular value of the voltage output of potentiometer and viceversa. Then the device is calibrated according to the readings recorded on the known surface (Ježko, 2014; Navidi et al., 1997; Scott et al., 2016).

\section{Experimentation, Results and Discussion}

The SLE is made to travel over know surfaces and tested several times and the observations are recorded in the form of graphs as shown in Fig. 3 and 4

The output voltage values of the potentiometer, stored in the memory of Arduino Uno are used to estimate the inclination of the surface at the various points in the area to be studied and thereby contour lines could be drawn with much easier than conventional methods from the 
results. Hence the instrument significantly reduces the human effort. The graphs are plotted from the values recorded from the experimentation. The experiments were conducted for $150 \mathrm{~cm}$ length track and the graphs are plotted in MATLAB.

In the graphs, it is observed that sometimes uncertain peaks are produced, this is due to the jerks when there is a sudden change of inclination of the surface. Due to the jerks the weight attached to the axis 2 may sometimes oscillate and during this period some of the uncertain output voltages of the potentiometer may be recorded, these observations don't correspond to the actual inclination of the surface at that point. Hence uncertain peaks and errors are found in the graphs (Jetko et al., 2011).

\section{Applications:}

- To find the elevations of given points with respective to an assumed datum and to establish points at a given elevation or at different elevations with respect to the assumed datum in the levelling process (Xeidakis, 1994)

- To determine the elevation of various points on the ground and at the same the horizontal positions for contouring process (Chrzanowski, 2014)

\section{Conclusion}

In this study, construction, working, experimentation and results of the surface level estimator are discussed. The objective of the design of the instrument is to reduce the human effort and time in surveying process. From the experimentation and results, we found that results obtained are not accurate and the uncertain peaks are formed in the graphs due to the jerks. Due to the jerks, sometimes the weight attached to the axis2 oscillates uncertainly and the output value of the potentiometer recorded during this period doesn't correspond to the actual inclination of the surfaces. Hence the errors are observed in the output graphs.

\section{Acknowledgement}

This research was supported by Maulana Azad National Institute of Technology. We would like to thank our institution for providing fund and expertise that greatly assisted the research, although they may not agree with all of the interpretations/conclusions of this paper.

\section{Funding Information}

We would like to thank Maulana Azad National Institute of Technology for provision of fund for prototyping and testing of surface level estimator.

\section{Author's Contributions}

The work is a product of the intellectual environment of both the authors; and both the authors have contributed in various degrees to the research concept and to the experiment design.

\section{Ethics}

The concept of SLE might result in the reduction of human work and Working manually for surveying of a land could be avoided. During large constructions, we see engineers working in the hot sun for surveying a land, those processes are tedious and time-consuming. Whereas SLE can take a higher position if more assistance is taken and provide work comfort for civil engineers.

\section{References}

Chrzanowski, A., 2014. Modern Surveying Techniques for Mining and Civil Engineering. In: Rock Testing and Site Characterization, Hudson, J.A. (Ed.), Pergamon Press, Oxford, ISBN-10: 1483297977, pp: 773-809.

Harris, H.C., C.C. Dahn, D.G. Monet and J.R. Pier, 1997. Trigonometric parallaxes of planetary nebulae. IAU Symp. 180: 40-45.

Jetko, J., 2011. Testing and calibration of surveying instruments and tools: Means to the quality increase of surveying works in construction. J. Interdisciplinary Res.

Ježko, J., 2014. Calibration of surveying instruments and tools-means to the quality increase of deformation measurements. J. Sustainable Min., 13: 17-22. DOI: $10.7424 /$ jsm 140404

Kyle, S.A., 1983. Surveying with non-levelled theodolites, Survey Rev., 27: 39-42. DOI: $10.1179 /$ sre.1983.27.207.39

Navidi, W., W.S. Murphy and W. Hereman, 1997. Statistical methods in surveying by trilateration. Comput. Stat. Data Anal., 27: 209-227. DOI: $10.1016 / \mathrm{S} 0167-9473(97) 00053-4$

Scott, D.N., D.J. Brogan, K.B. Lininger, D.M. Schook and E.E. Daugherty et al., 2016. Evaluating surveying instruments and methods in a steep channel. Geomorphology, 273: 236-243. DOI: 10.1016/j.geomorph.2016.08.020

Ustinova, N., V. Kala, T. Mill and A. Ellmann, 2012. Geodetic surveying studies for civil engineering students at Tallinn University of Technology. Geodesy Cartography, 38: 86-91. DOI: $10.3846 / 20296991.2012 .692213$

Xeidakis, G., 1994. Future directions of civil engineering education. Eur. J. Eng. Educ., 19: 141-146. DOI: $10.1080 / 03043799408923280$ 